\title{
Calculation of hyperfine structure constants for ytterbium
}

\author{
S G Porsev日, Yu G Rakhlina and M G Kozlov \\ Petersburg Nuclear Physics Institute, \\ Gatchina, St.-Petersburg district, 188350, RUSSIA
}

Submitted to: J. Phys. B: At. Mol. Opt. Phys.

\begin{abstract}
We calculate energies and hyperfine structure constants $A$ and $B$ for low-lying levels of ${ }^{173} \mathrm{Yb}$. The latter is treated as a two-electron atom. To account for valence-valence correlations the configuration interaction method is used. Valence-core correlations are accounted for within the many-body perturbation theory which is used to construct effective two-electron operators in the valence space.
\end{abstract}

\section{Introduction}

Two years ago a method for calculation of the low-lying energy levels of manyelectron atoms was proposed [1]. Calculations for $\mathrm{Tl}$ [1], $\mathrm{Ca}, \mathrm{Sr}, \mathrm{Ba}$ and $\mathrm{Yb}$ [2] and $\mathrm{Ba}$ [3] confirmed its effectiveness. Later this method was generalized for other observables, such as hyperfine structure (HFS) constants [4] and polarizabilities [5]. Within this method valence-valence correlations are treated non-perturbatively, while core-valence and core-core correlations are treated within the many-body perturbation theory (MBPT).

Here we report calculations for the HFS constants of the low-lying levels of ytterbium. Our interest to this atom is caused in part by an extremely large parity nonconserving (PNC) amplitude for the ${ }^{1} S_{0}\left(6 s^{2}\right) \rightarrow{ }^{3} D_{1}(5 d 6 s)$ transition. It was first suggested by DeMille [6] and later confirmed in [7, 8], that this amplitude is approximately 100 times larger than PNC amplitude for the $6 s \rightarrow 7 s$ transition in Cs and 10 times larger than that for the $6 p \rightarrow 7 p$ transition in $\mathrm{Tl}$. This makes $\mathrm{Yb}$ a very promising candidate for the future PNC experiment. Therefore, an accurate atomic calculations for $\mathrm{Yb}$ are needed. Moreover, it is important to have a reliable estimate of the accuracy of such calculations. PNC amplitude is very sensitive to the behavior of the wave function in the vicinity of the nucleus. Of course, the same applies to HFS constants. That makes HFS calculations very important for the future analysis of the theoretical accuracy for the PNC amplitude.

We also have a more general interest in ytterbium as an atom with the relatively shallow $4 f$ core shell. For such an atom it is not obvious at all, that core-valence correlations can be treated by means of the MBPT. In the earlier paper [2] we have

$\dagger$ E-mail: porsev@thd.pnpi.spb.ru 
shown that the method works for the low-lying energy levels and here we extend our analysis to the HFS constants. We also show here, that with some caution it is possible to calculate HFS constants of the levels, which lie above the core excitation threshold.

In the next section of the paper we define effective operators for the valence electrons and briefly describe how MBPT can be used to calculate these operators (for more details see [1, 团). In section 3 we give some details of the calculations followed by the discussion of the results in section 4 .

\section{Effective operators for valence electrons}

At present there are several methods of calculations for many-electron atoms. For atoms with one electron above a closed core a conventional MBPT is quite effective (see, for example, [9]). For atoms with several valence electrons the multi configurational Hartree-Fock (MCHF) method is often used [10]. Lately the coupledclaster method became very popular [11, 12, 13]. All these methods were used to calculate HFS constants of different atoms (see, for example, 14, 15, 16, 17, 18, 19]).

The most complicated problem in precise atomic calculations is associated with the necessity to treat valence-valence correlations, core-valence, and core-core correlations. The former are usually too strong to be treated perturbatively, while other types of correlations can not be effectively treated within non-perturbative techniques, such as MCHF or CI method.

Therefore, it is natural to try to combine MBPT with one of the non-perturbative methods. In [1] it was suggested to use MBPT in order to construct an effective Hamiltonian for valence electrons. After that, Schrödinger equation for valence electrons is solved within CI approach. That allows to find the low-lying energy levels. In order to calculate other atomic observables, one need to construct corresponding effective operators for valence electrons [4]. The latter effectively account for the corevalence and core-core correlation, which are of particular importance for such singular operators as HFS ones.

Suppose, that we are interested in atomic levels with energies $E_{i}-E_{0}<\varepsilon$, where $E_{0}$ is the ground state energy. In the first approximation we can assume that inner electrons with the Hartree-Fock energies $\left|\varepsilon_{n}\right| \gg \varepsilon$ form the core, which is described by the wave function

$$
\begin{aligned}
& \Psi_{\text {core }}=\left(N_{c} !\right)^{-1 / 2} \operatorname{det}\left(\phi_{1}, \phi_{2}, \ldots \phi_{N_{c}}\right), \\
& h_{\mathrm{DF}} \phi_{n}=\varepsilon_{n} \phi_{n},
\end{aligned}
$$

where $h_{\mathrm{DF}}$ is the Dirac-Fock operator, and $N_{c}$ is the number of core electrons. Note, that this operator can include the field of some valence electrons as well. For example, it is natural to consider $\mathrm{Yb}$ as a two-electron atom with the core $\left[1 s^{2} \ldots 4 f^{14}\right]$, and operator $h_{\mathrm{DF}}$ can be calculated for configuration $1 s^{2} \ldots 4 f^{14} 6 s^{2}$ (so called, $V^{N_{-}}$ approximation, $\mathrm{N}$ is the number of electrons in the atom).

Let us say that many-electron wave function $\Psi$ belongs to the valence subspace if core electrons are in the state (1). We will denote projector operator on this subspace by $P$. Then, operator $Q=1-P$ will project on the subspace for which at least one of the core electrons is excited to one of the states $\phi_{n}$ with $n>N_{c}$.

One can show [1], that Schrödinger equation

$$
H \Psi=E \Psi
$$


is equivalent to the following equation in the $P$-subspace for the function $\Phi=P \Psi$ :

$$
\begin{aligned}
& (P H P+\Sigma(E)) \Phi=E \Phi, \\
& \Sigma(E)=P V^{\prime} R_{Q}(E) V^{\prime} P,
\end{aligned}
$$

where $V^{\prime}$ is the operator of the residual Coulomb interaction and $R_{Q}(E)$ is the Green's function in $Q$-subspace:

$$
\begin{aligned}
& V^{\prime}=H-H_{0}, \\
& R_{Q}(E)=Q \frac{1}{E-Q H Q} Q .
\end{aligned}
$$

Operator $H_{0}$ is defined in terms of one-electron operator (2):

$$
H_{0}=\sum_{i=1}^{N} h_{\mathrm{DF}}\left(\vec{r}_{i}\right)-W .
$$

The constant $W$ in the right hand side of this equation is introduced to compensate the double counting of the two-electron interaction in the sum. It can be fixed, for example, by the requirement:

$$
\left\langle\Psi_{\text {core }}\left|H_{0}\right| \Psi_{\text {core }}\right\rangle=\left\langle\Psi_{\text {core }}|H| \Psi_{\text {core }}\right\rangle,
$$

which implies, that

$$
W=W_{0} \equiv \sum_{i=1}^{N_{c}} \varepsilon_{i}-\left\langle\Psi_{\text {core }}|H| \Psi_{\text {core }}\right\rangle .
$$

It is also possible to use $W$ as a free parameter to match the energy spectrum. This subject is discussed in more details elsewhere [20].

Equations (3) - (7) yield:

$$
\Psi=\left(P+R_{Q}(E) V^{\prime} P\right) \Phi,
$$

The orthonormality condition $\left\langle\Psi_{i} \mid \Psi_{k}\right\rangle=\delta_{i, k}$ is approximately equivalent to the following condition for $\Phi$ :

$$
\left\langle\Phi_{i}\left|1-\partial_{E} \Sigma(\bar{E})\right| \Phi_{k}\right\rangle \approx \delta_{i, k},
$$

where $\bar{E} \approx\left(E_{i}+E_{k}\right) / 2$. Note, that only the last of equations (14) - (12) is approximate.

The operator in the left hand side of equation (14) plays the role of an effective Hamiltonian $H_{\text {eff }}$. Equations (田) - (7) allow to construct it with the help of conventional diagrammatic technique [1] 2], which is based on the following representation of the Green's function:

$$
\begin{aligned}
& R_{Q}(E)=R_{Q}^{0}(E)+R_{Q}^{0}(E) V^{\prime} R_{Q}(E), \\
& R_{Q}^{0}(E)=Q \frac{1}{E-Q H_{0} Q} Q .
\end{aligned}
$$

In particular, if we restrict ourselves to the second order MBPT, we have to replace $R_{Q}(E)$ in the right hand side of (5) by $R_{Q}^{0}(E)$.

Suppose now, that we know solutions of Equation (1) and we want to use them to calculate an observable $a$, which is associated with one-electron operator $A$ :

$$
a=\langle\Psi|A| \Psi\rangle \text {. }
$$

Let us define effective operator $A_{\text {eff }}$ so, that

$$
a=\left\langle\Phi\left|A_{\mathrm{eff}}\right| \Phi\right\rangle .
$$


The following approximation for $A_{\text {eff }}$ was suggested in [4]

$$
A_{\text {eff }} \approx P A P+P V^{\prime} R_{Q}^{0}(E) A_{\text {rpa }} P+P A_{\text {rpa }} R_{Q}^{0}(E) V^{\prime} P,
$$

where $A_{\text {rpa }}$ corresponds to the well-known random-phase approximation (RPA).

Note, that only the first order MBPT corrections are completely included in (17). Some second order corrections, the, so-called, structural radiation, as well as many higher-order corrections, are omitted. Nevertheless, this approximation accounts for several most important MBPT corrections to all orders. Diagrammatic representation of Equation (17) can be found in [1.

\section{Hyperfine structure calculations}

We use basis representation for diagrams for effective Hamiltonian and for effective HFS operators. That implies that all sums over intermediate states run over some finite set of one-electron states. The latter should effectively account for both discrete and continuous part of the spectrum of Dirac-Fock operator (2).

In this calculation basis set included 167 four-component orbitals: $1-23 s, 2-22 p$, $3-22 d, 4-15 f, 5-15 g$ and $6-13 h$. Core orbitals as well as valence orbitals $6 s, 7 s, 6 p, 7 p$, and $5 d, 6 d$ were obtained by solving Dirac-Fock equations for appropriate configurations, while higher virtual orbitals were formed, as described in [21, 22, 1, 何. After that operator (2) was diagonalized in order to have quasi-Dirack-Fock basis set.

This basis set was used to solve RPA equations for HFS operators and to calculate diagrams for effective Hamiltonian and effective HFS operators. After that, the solution of Equation (位) was found in the CI approximation. On this stage it was possible to truncate our basis set to $7-15 s, 6-15 p, 5-14 d, 5-10 f$ and $5-7 g$ orbitals. We made complete CI on this shorter basis set.

We calculated effective Hamiltonian $H_{\text {eff }}$ in the second order of MBPT. That means, that zero order approximation (14) for the Green's function was used in (5). The choice of the constant $W$ suggested by Equation (10) resulted in some underestimation of the binding energy for levels of the configuration $5 d 6 s$ and for ${ }^{1} P_{1}^{o}$ level of the configuration $6 s 6 \mathrm{p}$. For these levels $W=W_{0}+0.4$ au was used. Our final results for the ground state are given in table 1 and for several low-lying levels in table 2 .

On the next stage we used corresponding wave functions to calculate HFS

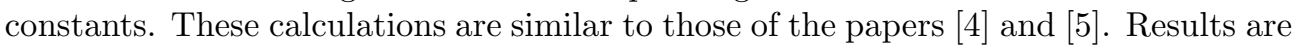
given in tables 3 and 1 . In analysis of the theoretical accuracy, which is done in the next section, it is very important to know the scale of different contributions to the final answer. Therefore, in tables 3 and 1 we give results of the Dirac-Fock calculation, of the conventional two-electron CI and of the two-electron CI with effective Hamiltonian. Final values include effective operator corrections (17) and normalization correction (12). The latter usually decreases the answer by $1 \%-2 \%$.

\section{Discussion}

We have pointed out above, that the effective Hamiltonian can be safely used only for the energy levels below the core excitation threshold. For Yb this threshold lies at $23189 \mathrm{~cm}^{-1}$ above the ground state and many of the levels from the table 2 lie higher. Nevertheless, theoretical spectrum is quite good up to the level ${ }^{1} \mathrm{P}_{1}^{o}(6 s 7 p)$, which appears $1634 \mathrm{~cm}^{-1}$ below its experimental position. This huge discrepancy can 
be attributed to interaction with the $J=1$ level at $38422 \mathrm{~cm}^{-1}$, which is supposed to belong to the configuration $4 f^{13} 5 d^{2} 6 s$ [23]. This level can also interact with ${ }^{3} \mathrm{P}_{1}^{o}(6 s 7 p)$, what makes calculations for this level less reliable. For other levels from the table 2 there are no close levels with the same $J$ and the same parity, which correspond to the excitations from the $4 f$-shell.

The perturber levels, discussed above, correspond to the poles of the Green's function $R_{Q}(E)$ and, thus, to the poles of the operator $\Sigma(E)$. As long as we use approximate Green's function (14) instead of the exact one, the poles of $\Sigma(E)$ are shifted from the physical poles. In order to use effective Hamiltonian, we have to keep far enough from the poles of the exact Green's function and from the poles of the approximate Green's function. As we go further up above the core excitation threshold, both sets of poles become denser. For this reason, it is hardly possible to use effective operator technique there.

Let us now proceed to the calculations of HFS constants presented in the tables 3 and 4 . In these tables the first two rows correspond to the non-perturbative part of the calculation, while two lower rows include MPBT corrections associated with the effective Hamiltonian and the effective HFS operators. One can see, that average MBPT contribution is about 30\% for magnetic constant $A$ and about $40 \%$ for electric quadrupole constant $B$. Thus, theoretical accuracy for the constant $A$ is normally higher. Indeed, we have neglected higher order corrections to the effective Hamiltonian and to the effective HFS operators, that can be justified only if MBPT corrections are small.

For 6 levels $\left({ }^{3} \mathrm{D}_{2}(5 d 6 s),{ }^{3} \mathrm{D}_{3}(5 d 6 s),{ }^{3} \mathrm{~S}_{1}(6 s 7 s),{ }^{3} \mathrm{D}_{1}(6 s 6 d),{ }^{3} \mathrm{D}_{3}(6 s 6 d)\right.$, and $\left.{ }^{3} \mathrm{P}_{2}^{o}(6 s 7 p)\right)$ MBPT corrections to the constant $A$ are less than $25 \%$. For all these levels the difference between the theory and experiment is within $3 \%$. For the constant $B$ the smallest MBPT corrections (about 35\%) correspond to levels ${ }^{3} \mathrm{D}_{1}(6 s 6 d)$ and ${ }^{3} \mathrm{P}_{1}^{o}(6 s 6 p)$, where we have agreement with the experiment within $2 \%$. Constant $B$ for ${ }^{3} \mathrm{~S}_{1}(6 s 7 s)$ - state differs from zero only because of the configuration interaction.

On the other hand, when MBPT corrections are $40 \%$ or more, the accuracy of the theory becomes uncertain. The most striking discrepancy with the experiment takes place for levels ${ }^{1} \mathrm{D}_{2}(5 d 6 s),{ }^{3} \mathrm{D}_{2}(6 s 6 d)$ and ${ }^{1} \mathrm{P}_{1}^{o}(6 s 6 p)$. For all of them MBPT corrections appear to be $40 \%-50 \%$.

The only exception of this rule is the level ${ }^{3} \mathrm{P}_{1}^{o}(6 s 7 p)$, where MBPT corrections for both constants are about $30 \%$, but even the sign of the constant $B$ differs from the experiment. As we pointed out above, this level can interact with the perturber level $4 f^{13} 5 d^{2} 6 s(J=1)$, which is located at $38422 \mathrm{~cm}^{-1}$. Constant $B$ for this level is relatively small and even small admixture of the perturber can result in the change of its sign, while the larger constant $A$ should be less affected by such a mixing.

\section{Conclusion}

In this paper we have checked applicability of the CI+MBPT method for ytterbium. The first core excitation here lies only $23189 \mathrm{~cm}^{-1}$ above the ground state, which seems to restrict us to only few lowest levels. Actually, the method works quite well up to $38930 \mathrm{~cm}^{-1}$, where the first significantly perturbed level arise.

When the method is used for calculations of HFS constants, the accuracy of calculations depends on the scale of MBPT corrections. When total MBPT correction contributes less than $25 \%$ to the value of the constant, the accuracy appears to be better than 3\%. On the contrary, when MBPT corrections account for more than 
$40 \%$ of the theoretical value, the accuracy becomes uncertain. In this case one can use MBPT correction only as a rough estimate of the theoretical error. We expect, that similar relation holds for other one-electron operators as well.

For the studied levels of $\mathrm{Yb}$ the average MBPT correction is approximately $30 \%$ for the constant $A$ and $40 \%$ for the constant $B$. Therefore, precision calculations of the constant $A$ are possible for the majority of levels, while precision calculations of the constant $B$ are possible only as exception.

\section{Acknowledgments}

This work was supported in part by Russian Foundation for Basic Research, Grant No 98-02-17663. SP is grateful to St. Petersburg government for the financial support. MK was supported by Engineering and Physical Sciencies Research Council.

\section{References}

[1] Dzuba V A, Flambaum V V, and Kozlov M G 1996 JETP Lett. 63 844; Phys. Rev. A 543948

[2] Kozlov M G and Porsev S G 1997 Sov. Phys.-JETP 111838

[3] Dzuba V A and Johnson W R 1998 Phys. Rev. A 572459

[4] Dzuba V A, Kozlov M G, Porsev S G and Flambaum V V 1998 Sov. Phys.-JETP (in press)

[5] Kozlov M G and Porsev S G 1998 Euro. Phys. Journ. D (in press)

[6] DeMille D 1995 Phys. Rev. Lett. 744165

[7] Porsev S G, Rakhlina Yu G and Kozlov M G 1995 JETP Lett 61459

[8] Das B P 1997 Phys. Rev. A 561635

[9] Lindgren I and Morrison J 1985 Atomic Many-Body Theory, 2nd ed. (Springer-Verlag, Berlin)

[10] Grant I P and Quiney H M 1988 Adv. At. Mol. Phys. 2337

[11] Blundell S A, Johnson W R and Sapirstein J 1991 Phys. Rev. A 433407

[12] Illibaev E and Kaldor U 1993 Phys. Rev. A 47137

[13] Illibaev E, Kaldor U and Ishikava Y 1994 Phys. Rev. A 49 1724; 50 1121; 51225

[14] Lindgren I 1984 Rep. Prog. Phys. 47345

[15] Dzuba V A, Flambaum V V, Silvestrov O P and Sushkov O P 1987 J. Phys. B: At. Mol. Opt. Phys. 201399

[16] Olsson T, Rosen A, Fricke B and Torbohm G 1988 Phys. Scr. 37730

[17] Beck D R and Datta D 1993 Phys. Rev. A 48182

[18] Jönsson P and Froese Fisher C 1993 Phys. Rev. A 484113

[19] Mårtensson-Pendrill A-M 1995 Phys. Rev. Lett. 742184

[20] Kozlov M G and Porsev S G 1999Optics and Spectroscopy (in press)

[21] Bogdanovich P and Žukauskas G 1983 Sov. Phys. Collection 2313

[22] Bogdanovich P, Žukauskas G and Šandžiuvienè S 1984 Sov. Phys. Collection 2420

[23] Martin W C, Zalubas R, and Hagen L 1978 Atomic Energy Levels - The Rare Earth Elements, NSRDS-NBS 60, National Bureau of Standards, Washington D.C.

[24] Töpper O, (private communication); Töpper O, Guthöhrlein G H, and Hillermann P 1997 Abstracts of 29 EGAS (Berlin) 233

[25] Berends R W and Maleki L 1992 J. Opt. Soc. Am. B 9332

[26] Jin W-G et al 1991 Journ. Phys. Soc. Jpn. 602896

[27] Clark D L, Cage M E, Lewis D E and Greenless G W 1979 Phys. Rev. A 20239

[28] Budick B and Snir J 1969 Phys. Rev. 17818

[29] Dzuba V A, Flambaum V V and Sushkov O P 1989 Phys. Lett. A 141147 
Calculation of hyperfine structure constants for ytterbium

Table 1. Two-electron energies $E_{\mathrm{val}}$ of the $\mathrm{Yb}$ ground state in different approximations (au). MBPT corrections were calculated with $W=W_{0}$ (see Equation (10)).

\begin{tabular}{|c|c|c|c|c|}
\hline $\mathrm{DF}^{\mathrm{a}}$ & $\mathrm{MBPT}^{\mathrm{b}}$ & $\mathrm{CI}^{\mathrm{C}}$ & $\mathrm{CI}+\mathrm{MBPT}^{\mathrm{d}}$ & Experiment $\mathrm{e}^{\mathrm{e}}$ \\
\hline 0.606810 & 0.654308 & 0.632398 & 0.677601 & 0.677584 \\
\hline
\end{tabular}

Table 2. The low-lying levels of $\mathrm{Yb}$ in $V^{N}$ approximation. $E_{\mathrm{val}}$ is the twoelectron energy and $\Delta$ is the interval from the ground state. The multiplet splittings are given in parentheses. Levels marked with $\dagger$ were calculated with $W=W_{0}+0.4 \mathrm{au}$ and the levels marked with $\ddagger$ were calculated with $W=$ $W_{0}+0.1 \mathrm{au}$.

\begin{tabular}{|c|c|c|c|}
\hline \multirow[b]{2}{*}{ Level } & \multicolumn{2}{|c|}{ Theory } & \multirow{2}{*}{$\Delta \begin{array}{l}\text { Exper. }^{\mathrm{a}} \\
\left(\mathrm{cm}^{-1}\right)\end{array}$} \\
\hline & $E_{\mathrm{val}}(\mathrm{au})$ & $\Delta\left(\mathrm{cm}^{-1}\right)$ & \\
\hline \multicolumn{4}{|c|}{ Even states } \\
\hline${ }^{3} \mathrm{D}_{1}(5 d 6 s) \dagger$ & -0.566086 & 24441 & 24489 \\
\hline${ }^{3} \mathrm{D}_{2}(5 d 6 s) \dagger$ & -0.564919 & $24697(256)$ & $24752(263)$ \\
\hline${ }^{3} \mathrm{D}_{3}(5 d 6 s) \dagger$ & -0.562415 & $25247(550)$ & $25271(519)$ \\
\hline${ }^{1} \mathrm{D}_{2}(5 d 6 s) \dagger$ & -0.551500 & 27642 & 27678 \\
\hline${ }^{3} \mathrm{~S}_{1}(6 s 7 s)$ & -0.529514 & 32501 & 32695 \\
\hline${ }^{1} \mathrm{~S}_{0}(6 s 7 s)$ & -0.521725 & 34210 & 34351 \\
\hline${ }^{3} \mathrm{D}_{1}(6 d 6 s)$ & -0.496053 & 39845 & 39809 \\
\hline${ }^{3} \mathrm{D}_{2}(6 d 6 s)$ & -0.495902 & $39877(33)$ & $39838(29)$ \\
\hline${ }^{3} \mathrm{D}_{3}(6 d 6 s)$ & -0.495384 & $39991(114)$ & 39966 (128) \\
\hline${ }^{1} \mathrm{D}_{2}(6 d 6 s)$ & -0.494733 & 40135 & 40062 \\
\hline \multicolumn{4}{|c|}{ Odd states } \\
\hline${ }^{3} \mathrm{P}_{0}^{o}(6 s 6 p) \ddagger$ & -0.598858 & 17282 & 17288 \\
\hline${ }^{3} \mathrm{P}_{1}^{o}(6 s 6 p) \ddagger$ & -0.595599 & $17997(715)$ & $17992(704)$ \\
\hline${ }^{3} \mathrm{P}_{2}^{o}(6 s 6 p) \ddagger$ & -0.587613 & $19750(1753)$ & $19710(1718)$ \\
\hline${ }^{1} \mathrm{P}_{1}^{o}(6 s 6 p) \dagger$ & -0.563354 & 25074 & 25068 \\
\hline${ }^{3} \mathrm{P}_{0}^{o}(6 s 7 p)$ & -0.504463 & 38000 & 38091 \\
\hline${ }^{3} \mathrm{P}_{1}^{o}(6 s 7 p)$ & -0.504096 & $38080(80)$ & $38174(83)$ \\
\hline${ }^{3} \mathrm{P}_{2}^{o}(6 s 7 p)$ & -0.502388 & $38455(375)$ & $38552(378)$ \\
\hline${ }^{1} \mathrm{P}_{1}^{o}(6 s 7 p)$ & -0.500224 & 38930 & 40564 \\
\hline
\end{tabular}


Table 3. Magnetic dipole $(A)$ and electric quadrupole $(B)$ hyperfine structure constants of low-lying even-parity levels for ${ }^{173} \mathrm{Yb}$. The electric quadrupole moment is taken to be $2.80 \mathrm{~b}$.

\begin{tabular}{|c|c|c|c|c|c|c|c|c|c|}
\hline & $\begin{array}{c}{ }^{3} D_{1} \\
(5 d 6 s)\end{array}$ & $\begin{array}{c}{ }^{3} D_{2} \\
(5 d 6 s)\end{array}$ & $\begin{array}{c}{ }^{3} D_{3} \\
(5 d 6 s)\end{array}$ & $\begin{array}{c}{ }^{1} D_{2} \\
(5 d 6 s)\end{array}$ & $\begin{array}{c}{ }^{3} S_{1} \\
(6 s 7 s)\end{array}$ & $\begin{array}{c}{ }^{3} D_{1} \\
(6 s 6 d)\end{array}$ & $\begin{array}{c}{ }^{3} D_{2} \\
(6 s 6 d)\end{array}$ & $\begin{array}{c}{ }^{3} D_{3} \\
(6 s 6 d)\end{array}$ & $\begin{array}{c}{ }^{1} D_{2} \\
(6 s 6 d)\end{array}$ \\
\hline \multicolumn{10}{|c|}{$A(\mathrm{MHz})$} \\
\hline DF & 447 & -200 & -348 & -18 & -1225 & 490 & -206 & -337 & 28 \\
\hline CI & 443 & -288 & -348 & 62 & -1489 & 633 & -468 & -437 & 238 \\
\hline$H_{\text {eff }}$ & 588 & -398 & -469 & 105 & -1910 & 830 & -677 & -567 & 380 \\
\hline Total & 596 & -351 & -420 & 131 & -1938 & 838 & -683 & -569 & 392 \\
\hline Exper. $^{\mathrm{a}}$ & $563(1)$ & $-362(2)$ & $-430(1)$ & $100(18)$ & $-1879(10)$ & $818.7(4)$ & $-732.5(4)$ & $-559.9(5)$ & $438.5(4)$ \\
\hline \multicolumn{10}{|c|}{$B(\mathrm{MHz})$} \\
\hline DF & 151 & 206 & 353 & 379 & 0 & 32 & 45 & 76 & 81 \\
\hline CI & 156 & 229 & 368 & 676 & 0.3 & 38 & 55 & 92 & 115 \\
\hline$H_{\text {eff }}$ & 219 & 314 & 492 & 693 & 0.2 & 38 & 62 & 92 & 115 \\
\hline Total & 290 & 440 & 728 & 1086 & 0.2 & 58 & 96 & 150 & 184 \\
\hline Exper. $^{a}$ & $335(1)$ & $482(22)$ & $909(29)$ & 1115(89) & $<3(18)$ & $59.3(2)$ & $52.5(2)$ & 139.6(3) & $142.2(2)$ \\
\hline
\end{tabular}

${ }^{a}$ HFS constants for configuration $5 d 6 s$ were measured in $\left[24\right.$, for ${ }^{3} \mathrm{~S}_{1}$-state in 25$]$, and for configuration $6 s 6 d$ in 26

Table 4. Magnetic dipole $(A)$ and electric quadrupole $(B)$ hyperfine structure constants of low-lying odd-parity levels for ${ }^{173} \mathrm{Yb}$.

\begin{tabular}{|c|c|c|c|c|c|}
\hline & $\begin{array}{c}{ }^{3} P_{1}^{o} \\
(6 s 6 p)\end{array}$ & $\begin{array}{c}{ }^{3} P_{2}^{o} \\
(6 s 6 p)\end{array}$ & $\begin{array}{c}{ }^{1} P_{1}^{o} \\
(6 s 6 p)\end{array}$ & $\begin{array}{c}{ }^{3} P_{1}^{o} \\
(6 s 7 p)\end{array}$ & $\begin{array}{c}{ }^{3} P_{2}^{o} \\
(6 s 7 p)\end{array}$ \\
\hline \multicolumn{6}{|c|}{$A(\mathrm{MHz})$} \\
\hline $\begin{array}{l}\mathrm{DF} \\
\mathrm{CI} \\
H_{\text {eff }} \\
\text { Total } \\
\text { Exper. }\end{array}$ & $\begin{array}{c}-664 \\
-765 \\
-1075 \\
-1094 \\
-1094.0(7)^{\mathrm{a}} \\
-1094.2(6)^{\mathrm{d}}\end{array}$ & $\begin{array}{l}-527 \\
-556 \\
-741 \\
-745 \\
-738^{\mathrm{b}}\end{array}$ & $\begin{array}{c}-25 \\
98 \\
187 \\
191 \\
59^{\mathrm{b}}\end{array}$ & $\begin{array}{c}-667 \\
-1044 \\
-1468 \\
-1488 \\
-1144(1)^{\mathrm{c}}\end{array}$ & $\begin{array}{c}-507 \\
-666 \\
-862 \\
-871 \\
-854(2)^{c}\end{array}$ \\
\hline \multicolumn{6}{|c|}{$B(\mathrm{MHz})$} \\
\hline $\begin{array}{l}\mathrm{DF} \\
\mathrm{CI} \\
H_{\text {eff }} \\
\text { Total } \\
\text { Exper. }\end{array}$ & $\begin{array}{c}-454 \\
-533 \\
-633 \\
-822 \\
-826.5(1)^{\mathrm{a}} \\
-827.2(5)^{\mathrm{d}}\end{array}$ & $\begin{array}{c}715 \\
860 \\
1013 \\
1335 \\
1312^{\mathrm{b}}\end{array}$ & $\begin{array}{c}811 \\
428 \\
642 \\
848 \\
605^{\mathrm{b}}\end{array}$ & $\begin{array}{c}-90 \\
-80 \\
-82 \\
-111 \\
12(4)^{\mathrm{c}}\end{array}$ & $\begin{array}{c}150 \\
163 \\
192 \\
264 \\
267(25)^{\mathrm{c}}\end{array}$ \\
\hline
\end{tabular}

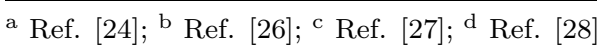

